\title{
Theory of the Polarized Fluorescence Experiment
}

\author{
Eckart Frehland \\ Fachbereich Physik der Universität Konstanz, Germany \\ (Z. Naturforsch. 30 a, 1241-1246 [1975] ; received July 9, 1975)
}

\begin{abstract}
The static polarized fluorescence experiment is investigated for the most general case of arbitrary orientation distributions of transition moments in the fluorescent sample and arbitrary rearrangements of transition moments during the excited fluorescent state. It is shown that all available information is contained in a tensor of fourth order. The maximum number of independent components of this tensor is 36 . Hence in the general case 36 polarized intensities must be measured in order to utilize the maximum available information. For special symmetries of orientation distributions this number is drastically reduced. In the case of vanishing rearrangement it is 15, for planar arrays (axial symmetry) not exceeding 11 and for spherical symmetry only 2 . The results may be extended to the time-dependent polarized fluorescence experiment.
\end{abstract}

\section{Introduction}

It was Francis Perrin ${ }^{1,2,3}$ who first investigated the influence of Brownian rotation of macromolecules in solutions on fluorescence polarization. Since that time this theory has been confirmed and extended to anisotropic rotations and time-dependent depolarization by several authors (see e.g. ${ }^{4-10}$ ). Generally, from the polarized fluorescence experiment with fluorescent molecules in solutions one can get information about the mobility of the fluorescent molecules.

On the other hand fluorescence polarization represents a possibility of measuring preferred orientations. E.g. orientations of amorphous polymers have been determined with the use of fluorescence polarization experiments by Nishijima et al. ${ }^{11,12}$. The advantage of fluorescence polarization experiments compared with other methods of studying orientations such as birefringence and dichroism measurements is, that generally they contain more information ${ }^{13}$.

More recently, the interest in fluorescence polarization for the study of mobility and orientation of pigment molecules in biological and lipid bilayer model membranes has increased (see among others $\left.{ }^{14-17}\right)$. The theoretical analysis of such experiments is more complicated, because the influence of both, orientation and mobility (or more exactly of rearrangements of transition moments during the excited state) must be considered.

The problem how to draw a conclusion from the results of the static polarized fluorescence experiment on the properties of the fluorescent sample leads

Reprint requests to Dr. E. Frehland, Fachbereich Physik der Universität Konstanz, D-7750 Konstanz, Postfach 7733 . directly to the central question of this paper: which and how much independent information is in principal available? The answer to this question is important for an economic performance of the theoretical analysis as well as for a minimization of the experimental work without loozing information. The question has been answered by the work of Desper and Kimura ${ }^{13}$ under the restricting assumptions that the transition moments of absorption and emission are parallel, i.e. no rearrangement occurs, and that the orientation distribution of transition moments is biaxially symmetric. For this case they could show that all information is contained in six polarized intensities.

In this paper we shall solve the problem for the most general case of arbitrary orientation distributions of transition moments and for arbitrary rearrangements of transition moments during the excited fluorescent state. The processes underlying these rearrangements, which cause a nonparallelity of transitions of absorption and emission, must not be specified. They may be rotational motion of the fluorescent molecules, energy transfer between fluorescent molecules, nonvanishing angles between the transition moments within the molecules etc. All available information is contained in a tensor of fourth order. This "fluorescence tensor" has 36 independent components. Hence 36 independent polarized intensities must be measured in order to utilize all available information. In case special symmetries or other properties of the fluorescent probe are known, the number of independent quantities is reduced. Special examples shall be discussed. The results may also be applied for time-dependent fluorescence (de-) polarization. In this case the fluorescence tensor becomes time-dependent. 


\section{The Static Polarized Fluorescence Experiment}

Strongly simplified the fluorescence process may be described by

absorption $\underset{\text { of transition moments }}{\text { rearrangement (reorientation) }}$ emission.

A schematic picture of the polarized fluorescence experiment is given in Fig. 1: A monochromatic

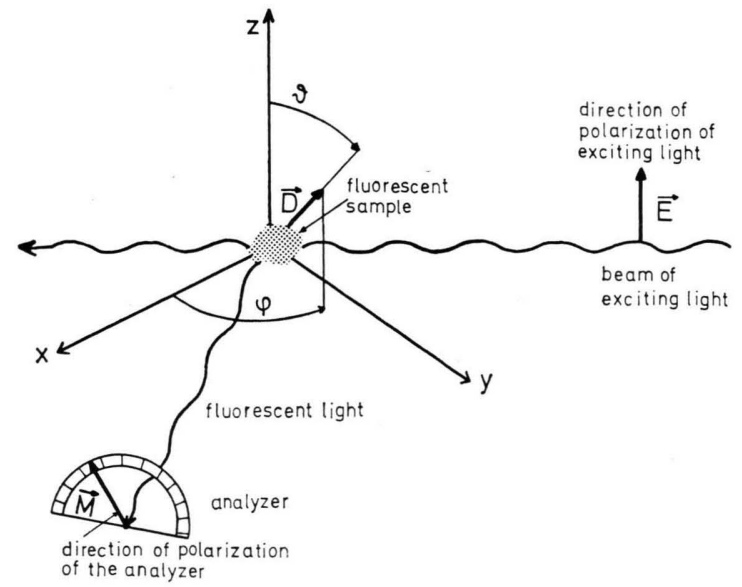

Fig. 1. Geometry of the polarized fluorescence experiment. $(\overrightarrow{\mathrm{E}}, \overrightarrow{\mathrm{D}}, \overrightarrow{\mathrm{M}}=\boldsymbol{E}, \boldsymbol{D}, \boldsymbol{M}$.

linearly polarized light beam excites a fluorescent probe. The intensity of fluorescent light, emitted by this probe, is measured in arbitrary direction through polarizing analyzers. Cartesian coordinates $(x, y, z)$ and spherical polar angles $\vartheta, \varphi$ are introduced. The directions of polarization of incident light and of the analyzer are given by normalized vectors $\boldsymbol{E}, \boldsymbol{M}$ respectively $\left(\boldsymbol{E}^{2}=\boldsymbol{M}^{2}=1\right)$. The direction of dipole transition moments is given by normalized vectors $\boldsymbol{D}(\vartheta, \varphi)$ [see Eq. (4) ].

The following basic assumptions are used:

I. The number of fluorescent molecules in the excited state is small compared with the total number of fluorescent molecules.

II. Intensity and directional dependence of absorption and emission are described by the classical theory of oscillating dipoles.

III. The extension of the fluorescent area is small compared with the distance to the detecting system (far-field assumption).

IV. Existence of a stationary equilibrium distribution $F_{\mathrm{A}}$ of transition moments of absorption.

V. Existence of a stationary (fluorescence) intensity of emission $I_{\mathrm{E}}$.
The last two assumptions guarantee that the polarized fluorescence intensities in arbitrary directions are static if the fluorescent probe is excited by light of constant intensity.

In the following three different distributions shall be used: the orientation distribution $F_{\mathrm{A}}(\vartheta, \varphi)$ of transition moments of absorption, the intensity of absorption $I_{\mathrm{A}}(\vartheta, \varphi)$ and the intensity of emission $I_{\mathrm{E}}(\vartheta, \varphi)$. All three distributions are defined as densities on the unit sphere. Under the assumptions I, II the intensity of absorption is proportional to $F_{\mathrm{A}}$ and $\cos ^{2} \Psi$, where $\Psi$ is the angle between the direction of polarization of incident light and the direction $\boldsymbol{D}(\vartheta, \varphi)$ of transition moments of absorption in position $(\vartheta, \varphi)$.

$$
I_{\mathrm{A}}=P_{\mathrm{A}} \cdot F_{\mathrm{A}}(\vartheta, \varphi) \cdot \cos ^{2} \Psi(\mathbf{E}, \vartheta, \varphi),
$$

$F_{\mathrm{A}}(\vartheta, \varphi)$ is normalized to unity:

$$
\int \mathrm{d} \Omega F_{\mathrm{A}}=1, \quad \mathrm{~d} \Omega=\sin \vartheta \mathrm{d} \vartheta \mathrm{d} \varphi .
$$

The integration is taken over the unit sphere. The factor $P_{\mathrm{A}}$ depends on different quantities, e.g. the intensity of incident light, concentration of the fluorescent molecules and an apparatus constant. We do not specify this factor and shall use the reduced intensity $\bar{I}_{\mathrm{A}}$

$$
\bar{I}_{\mathrm{A}}=F_{\mathrm{A}} \cdot \cos ^{2} \Psi
$$

which is independent of the intensity of incident light $J$. If necessary, reduced quantities shall be marked by a bar $($ e.g. $I \rightarrow \bar{I})$. Because of assumption $I$ proportionality between $I_{\mathrm{A}}$ and $J$ can be assumed.

In case no rearrangement of transition moments takes place during the excited state the moments of absorption and emission are parallel and the intensity of emission $I_{\mathrm{E}}$ is proportional to $I_{\mathrm{A}}$. But generally a complex rearrangement of transition moments in the excited state must be considered. This rearrangement may be caused by complete different processes: First, the transition moments of absorption and emission must not be parallel within the fluorescent molecule. Second, energy transfer between fluorescent molecules may cause a significant rearrangement. Third, a rearrangement of transition moments may be done by rotational motions of the fluorescent molecules or their chromophoric part in the excited state.

Taking into account these or other possibilities of rearrangement from the intensity of absorption an intensity of emission $I_{\mathrm{E}}$ may be determined. Introducing the angle $\varkappa$ between a moment of emission 
in direction $\mathbf{D}(\vartheta, \varphi)$ and the direction of polarization $\boldsymbol{M}$ of the analyzer, the measured polarized fluorescence intensity $I_{\mathrm{M}}$ is given by integration over all directions with $I_{\mathrm{E}}$ as weighting function

$$
I_{\mathrm{M}}=P_{\mathrm{E}} \cdot \int \mathrm{d} \Omega I_{\mathrm{E}}(\vartheta, \varphi) \cos ^{2} \varkappa(\boldsymbol{M}, \vartheta, \varphi) .
$$

$x$ is the angle between a moment of emission in direction $\boldsymbol{D}$ and the polarization direction $\boldsymbol{M}$ of the analyzer. Omitting the factor of proportionality $P_{\mathrm{E}}$ the corresponding relation for the reduced intensities $\bar{I}_{\mathrm{E}}, \bar{I}_{\mathrm{M}}$ is :

$$
\bar{I}_{\mathrm{M}}=\int \mathrm{d} \Omega \bar{I}_{\mathrm{E}} \cos ^{2} \varkappa .
$$

\section{The Tensors of Absorption and Emission}

In the following vectors (and tensors) are alternatively written as quantities with indices:

$$
\boldsymbol{E} \rightarrow E_{\mathrm{a}}, \boldsymbol{D} \rightarrow D_{\mathrm{a}}, \boldsymbol{M} \rightarrow M_{\mathrm{a}}
$$

etc. Latin indices range over $1,2,3 . E_{1}$ is the $x$-component $\left(x^{1}=x\right), E_{2}$ the $y$-component $\left(x^{2}=y\right), E_{3}$ the $z$-component $\left(x^{3}=z\right)$ etc. The $x, y, z$-components of the normalized vectors $\boldsymbol{D}$ may be expressed by the angles $\vartheta, \varphi$ :

$$
\begin{aligned}
& D_{x}=D_{1}=\sin \vartheta \cos \varphi, \\
& D_{y}=D_{2}=\sin \vartheta \sin \varphi, \\
& D_{z}=D_{3}=\cos \vartheta .
\end{aligned}
$$

Regard the total absorbed intensity $\bar{A}$, which is given by integration of $\bar{I}_{\mathrm{A}}$ over the unit sphere. With $\cos \Psi=\boldsymbol{E} \cdot \mathbf{D}$ we get from $(1, \mathrm{c})$ :

$$
\bar{A}=\int \mathrm{d} \Omega\left(\sum_{a} E_{a} D_{a}\right)^{2} F_{\mathrm{A}} .
$$

With the symmetric matrix $\bar{A}_{a b}$ defined by

$$
\bar{A}_{a b}=\int F_{\mathrm{A}} D_{a} D_{b} \mathrm{~d} \Omega, \quad \bar{A}_{a b}=\overline{A_{b a}}
$$

from (5) follows immediately that $\bar{A}$ is given by $\bar{A}_{a b}$ through

$$
\bar{A}=\sum_{a, b} \overline{A_{a b}} E_{a} E_{b} .
$$

For arbitrary directions of excitation $\boldsymbol{E}$ the total absorbed intensity is determined by the 6 independent components of $\bar{A}_{a b} \cdot \bar{A}_{a b}$ has the properties of a tensor of second order. For introduction to tensor calcules see e.g. ${ }^{18}$. Hence the quantity $\bar{A}$ is a scalar, i.e. independent of the special choice of (cartesian) coordinates. We call $\bar{A}_{a b}$ the tensor of absorption. Because $\overline{A_{a b}}$ is symmetric, there exist principal axes $x^{*}, y^{*}, z^{*}$, with respect to which the non-diagonal elements of $\bar{A}_{a b}$ vanish. The 3 eigenvalues of
$\bar{A}_{a b}$ are the components $\overline{A_{a a}}$ in these coordinates $x^{*}, y^{*}, z^{*}$.

These results can be used in the polarized absorption experiment for measuring orientation distributions $F_{\mathrm{A}}$ : In the most general case, where no symmetries of the orientation distribution are known, all information is contained in six quantities. If the principal axes are known (3 informations), this number reduces to 3 . For axial symmetry and known principal axes it is 2 . The definition (6) of $\bar{A}_{a b}$ shows that with the polarized absorption experiment can be determined the multipole moments of the orientation distribution $F_{\mathrm{A}}$ up to the quadrupole moments. The monopole moment is just the (invariant) trace $\sum_{c} \bar{A}_{c c}$ of $\bar{A}_{a b}$, which in the reduced case is normalized to unity. The dipole moment and all further odd moments vanish, because orientations $\boldsymbol{D}$ and $-\boldsymbol{D}$ are undistinguishable and hence the relation

$$
F_{\mathrm{A}}(\vartheta, \varphi)=F_{\mathrm{A}}(\pi-\vartheta, \pi+\varphi)
$$

is valid. The tensor of the quadrupole moments $\stackrel{\mathrm{A}}{Q}_{a b}$ of $F_{\mathrm{A}}$ is defined by

$$
2 \stackrel{\mathrm{A}}{Q_{a b}}=\int F_{\mathrm{A}}\left(3 D_{a} D_{b}-\delta_{a b}\right) \mathrm{d} \Omega=3 \bar{A}_{a b}-\delta_{a b} .
$$

The process of emission may be treated in complete analogy if one replaces $F_{\mathrm{A}}, \boldsymbol{E}, \bar{A}$ by $I_{\mathrm{E}}, \boldsymbol{M}, \bar{I}_{\mathrm{M}}$ respectively. We introduce the second order tensor of emission $\bar{I}_{a b}$

$$
\bar{I}_{a b}=\int \bar{I}_{\mathrm{E}} D_{a} D_{b} \mathrm{~d} \Omega, \bar{I}_{a b}=\bar{I}_{b a} .
$$

The measured polarized fluorescence intensity $\bar{I}_{\mathrm{M}}$ is for arbitrary direction $\boldsymbol{M}$ of the analyzer:

$$
\bar{I}_{\mathrm{M}}=\sum_{a, b} \bar{I}_{a b} M_{a} M_{b} \text {. }
$$

Hence by the polarized fluorescence experiment are measured the multipole moments of the intensity of emission $I_{\mathrm{E}}$ up to the quadrupole moment.

Generally $\bar{I}_{a b}$ depends on the direction of polarization $\boldsymbol{E}$ of incident light. For given $\boldsymbol{E}$ the maximum possible number of independent quantities is 6 . The problem now arises, for which and for how many directions $\boldsymbol{E}$ the tensor of emission $\bar{I}_{a b}$ must be measured in the polarized fluorescence experiment, in order to get all possible information about orientation and rearrangement of transition moments. This problem will be solved in the next section.

\section{The Fluorescence Tensor}

Above we have mentioned different possibilities of rearrangement of transition moments during the 
excited state. The following is as general as possible and not related to a special process underlying this rearrangement. We introduce a function $F\left(\vartheta, \varphi, \vartheta^{\prime}, \varphi^{\prime}\right) \geqq 0$, relating two orientations $(\vartheta, \varphi)$ and $\left(\vartheta^{\prime}, \varphi^{\prime}\right)$ in the following way: if a transition moment of absorption in orientation between $(\vartheta, \varphi)$ and $(\vartheta+\mathrm{d} \vartheta, \varphi+\mathrm{d} \varphi)$ is excited then with a probability proportional to $F\left(\vartheta, \varphi, \vartheta^{\prime}, \varphi^{\prime}\right) \mathrm{d} \Omega \mathrm{d} \Omega^{\prime}$ this excitation induces an emission by a transition moment in orientation between $\left(\vartheta^{\prime}, \varphi^{\prime}\right)$ and $\left(\vartheta^{\prime}+\mathrm{d} \vartheta^{\prime}\right.$, $\left.\varphi^{\prime}+\mathrm{d} \varphi^{\prime}\right)$. If two, three ... transition moments in orientation $(\vartheta, \varphi)$ are excited, then the probability of emission in orientation $\left(\vartheta^{\prime}, \varphi^{\prime}\right)$ is greater by a factor two, three... In other words, the intensity of emission by transition moments in orientation $\left(\vartheta^{\prime}, \varphi^{\prime}\right)$ induced by excitation of absorption moments in orientation $(\vartheta, \varphi)$ is proportional to the intensity of absorption $I_{\mathrm{A}}(\vartheta, \varphi)$, i.e. proportional to the orientation distribution $F_{\mathrm{A}}(\vartheta, \varphi)$ and $\cos ^{2} \Psi(\boldsymbol{E}, \vartheta, \varphi)$ [see Equation (1)]. Essentially the described set up for $F\left(\vartheta, \varphi, \vartheta^{\prime}, \varphi^{\prime}\right)$ may be justified by the assumption I: Interactions between molecules in the excited state can be excluded, because their number is assumed to be small.

Hence for direction of polarization $\boldsymbol{E}$ of incident light the emitted polarized fluorescence intensity measured through an analyzer with polarization direction $\boldsymbol{M}$ is given by:

$$
\begin{gathered}
\bar{I}_{\mathrm{M}}=\int \mathrm{d} \Omega^{\prime} \cos ^{2} \varkappa\left(\boldsymbol{M}, \vartheta^{\prime}, \varphi^{\prime}\right) \\
\cdot\left[\int \mathrm{d} \Omega F\left(\vartheta, \varphi, \vartheta^{\prime}, \varphi^{\prime}\right) F_{\mathrm{A}}(\vartheta, \varphi) \cos ^{2} \Psi(\boldsymbol{E}, \vartheta, \varphi)\right], \\
\iint \mathrm{d} \Omega \mathrm{d} \Omega^{\prime} F=1 .
\end{gathered}
$$

By comparison with (2) follows

$$
\begin{aligned}
I_{\mathrm{E}}(\boldsymbol{E}, \vartheta, \varphi)=\int \mathrm{d} \Omega F\left(\vartheta, \varphi, \vartheta^{\prime}, \varphi^{\prime}\right) & \cdot F_{\mathrm{A}}(\vartheta, \varphi) \cos ^{2} \Psi(\boldsymbol{E}, \vartheta, \varphi) .
\end{aligned}
$$

The relation (12) suggests the introduction of the following four-indexed quantity $F_{a b c d}$ :

$$
\begin{aligned}
F_{a b c d}= & \int \mathrm{d} \Omega^{\prime} D_{a}\left(\vartheta^{\prime}, \varphi^{\prime}\right) D_{b}\left(\vartheta^{\prime}, \varphi^{\prime}\right) . \\
& \cdot\left[\int \mathrm{d} \Omega F\left(\vartheta, \varphi, \vartheta^{\prime}, \varphi^{\prime}\right) F_{\mathrm{A}}(\vartheta, \varphi) D_{c}(\vartheta, \varphi)\right. \\
& \left.\cdot D_{d}(\vartheta, \varphi)\right] .
\end{aligned}
$$

$F_{a b c d}$ satisfies the symmetry relations

$$
F_{a b c d}=F_{b a c d}=F_{a b d c} .
$$

For given $\boldsymbol{M}$ and $\boldsymbol{E}$ holds

$$
\bar{I}_{\mathrm{M}}=\sum_{a, b, c, d} M_{a} M_{b} F_{a b c d} E_{c} E_{d} .
$$

By comparison with (7), (11) we see that $F_{a b c d}$ contains the tensors of absorption and emission through

$$
\overline{\mathrm{A}}_{c d}=\sum_{a} F_{\text {aacd }}
$$

and

$$
\bar{I}_{a b}=\sum_{c, d} F_{a b c d} E_{c} E_{d} .
$$

$F_{a b c d}$ has the properties of a tensor of fourth order. As consequence the central relation (16) is independent of the special choice of (cartesian) coordinates. We call $F_{a b c d}$ the fluorescence tensor.

From the symmetry relations (15) follows, that the maximum number of independent components of $F_{a b c d}$ is 36 . This means that in the experiment 35 independent quantities are available being independent of the absolute intensities. For special symmetries of orientation distributions and special types of rearrangement of transition moments this number may be reduced drastically. Examples are discussed in the next section. By measurements of $I_{\mathrm{M}}$ in different directions of excitation and analyzer the components of $F_{a b c d}$ may be calculated by a system of linear equations according to (16). Excitation with circularly or elliptically polarized light yields no further information because these polarizations may be considered to be a superposition of different linear polarizations.

\section{Special Examples}

\section{a) Parallel transition moments of absorption and emission}

In case no rearrangement can take place during the excited state, the transition moments of absorption and emission are parallel and (14) simplifies to

$$
F_{a b c d}=\int \mathrm{d} \Omega F_{\mathrm{A}} D_{a} D_{b} D_{c} D_{d} .
$$

$F_{a b c d}$ is now totally symmetric:

$$
F_{a b c d}=F_{b a c d}=F_{a b d c}=F_{a c b d} .
$$

With (19) the maximum number of independent components of $F_{a b c d}$ is reduced to $15 . F_{a b c d}$ contains the even multipole moments of the distribution $F_{\mathrm{A}}$ up to the hexadecapole moment. As mentioned above [cf. (8)] the odd moments (dipole, octopole etc.) of $F_{\mathrm{A}}$ vanish. The hexadecapole $Q_{a b c d}$ of $F_{\mathrm{A}}$ is with (4) (in Cartesian coordinates) given by:

$$
\begin{aligned}
\stackrel{\mathrm{A}}{Q}_{a b c d}= & \int \mathrm{d} \Omega F_{\mathrm{A}} \cdot \frac{1}{8}\left\{\left(36 D_{a} D_{b} D_{c} D_{d}\right)-\right. \\
& -5\left(D_{a} D_{b} \delta_{c d}+D_{a} D_{c} \delta_{b d}+D_{a} D_{d} \delta_{b c}+\right. \\
& \left.+D_{b} D_{c} \delta_{a d}+D_{b} D_{d} \delta_{a c}+D_{c} D_{d} \delta_{a b}\right) \\
& \left.+\left(\delta_{a b} \delta_{c d}+\delta_{a c} \delta_{b d}+\delta_{a d} \delta_{b c}\right)\right\}
\end{aligned}
$$

with

$$
\delta_{a b}= \begin{cases}1 & \text { for } a=b \\ 0 & \text { for } a \neq b .\end{cases}
$$


The first bracket on the right hand of this relation is given by $F_{a b c d}$, the second is determined by the quadrupole tensor $\stackrel{\mathrm{A}}{a b}_{a b}$ and the third by the monopole moment of $F_{\mathrm{A}}$.

The number of independent components is further reduced for special symmetries of orientation distribution $F_{\mathrm{A}}$. Substituting $\vartheta, \varphi$ by $x, y, z \mathrm{D}_{2 \mathrm{~h}}$ symmetry (biaxial symmetry) is defined by

$$
\begin{aligned}
F_{\mathrm{A}}(x, y, z)=F_{\mathrm{A}}(-x, y, z) & =F_{\mathrm{A}}(x,-y, z) \\
& =F_{\mathrm{A}}(x, y,-z) .
\end{aligned}
$$

Then according to (20) only the six independent components $F_{a a b b}$ are nonvanishing. This is in agreement with the results of Desper and Kimura ${ }^{13}$ who derived the symmetric matrix $S_{a b}$ with the components

$$
S_{a b}=F_{a a b b}
$$

to be relevant for the analysis of the experiments.

\section{b) Axial symmetry of $F_{A}(\vartheta, \varphi)$}

In many cases, e.g. for fluorescent molecules in planar arrays ${ }^{14-17}$, the orientation distribution $F_{\mathrm{A}}$ is axially symmetric. If the axis of symmetry is known, it can be chosen to be the $z$-axis $(\vartheta=0)$. In these coordinates $F_{\mathrm{A}}$ is independent of $\varphi$. The tensor of absorption $\bar{A}_{a b}$ is diagonal and from (17 a) follows

$$
\sum_{a} F_{a a c d}=0 \text { for } c \neq d .
$$

The two eigenvalues $\bar{A}_{x x}, \bar{A}_{y y}$ are equal. By excitation $\boldsymbol{E}$ parallel to the $z$-axis $\left(E_{x}=E_{y}=0, E_{z}=1\right)$, perpendicular $\left(E_{x}=1, E_{y}=E_{z}=0\right)$ and in an intermediate direction (e.g. $E_{x}=E_{z}=1 / \sqrt{2}, E_{y}=0$ ) the components $F_{a b z z}, F_{a b x x}$ and $F_{a b x z}$ can be measured. The other components of $F_{a b c d}$ are not independent. For excitation parallel to the $z$-axis, the rearrangement must be independent of $\varphi$ and the tensor for emission $\bar{I}_{a b}$ is in diagonal form with $\bar{I}_{x x}=\bar{I}_{y y}$. Hence $F_{a b z z}=0$ for $a \neq b$ and $F_{x x z z}=F_{y y z z}: 2 \mathrm{com}$ ponents of $F_{a b z z}$ are independent. For excitation perpendicular one eigendirection of $\bar{I}_{a b}$ is the $z$-axis and $F_{a z x x}=0$ for $z \neq x^{a}$. Hence 4 components of $F_{a z x x}$ are independent. Finally excitation in intermediate direction $\left(E_{x}=E_{z}=1 / \sqrt{ } 2\right)$ determines $F_{a b x y}$ according to $(17 \mathrm{~b})$ through:

$$
\bar{I}_{a b}=\frac{1}{2}\left(F_{a b x x}+F_{a b z z}\right)+F_{a b x z} .
$$

Because of relation (22) only 5 components of $F_{a b x z}$ are independent.
Therefore the maximum number of independent polarized intensities, i.e. components of $F_{a b c d}$, which must be measured in the axial symmetric case, is not exceeding $2+4+5=11$. Often for special assumptions about the rearrangement this number can be further reduced.

\section{c) Isotropic distributions}

As a last example we consider isotropic orientation distributions. Isotropic distributions are given for molecules in solutions or for molecules incorporated into spherical membranes. In the classical theory of fluorescence depolarization only one degree of polarization (or alternatively the molecular anisotropy) is used for the analysis of the polarized fluorescence experiment, i.e. two absolute intensities polarized parallely and perpendicularly to the direction of excitation $\boldsymbol{E}$.

Indeed it may easily be verified that generally in the isotropic case the number of independent components of the fluorescence tensor $F_{a b c d}$ is two. 'Isotropic case' means that not only the stationary distribution $F_{\mathrm{A}}$ is isotropic but also the surrounding medium. No direction is marked out concerning the processes of absorption, rearrangement and emission. Because the orientation distribution $F_{\mathrm{A}}$ does not depend on $\vartheta, \varphi$ the tensor of absorption is in diagonal form for arbitrary choice of (cartesian) coordinates $x, y, z$ and the three eigenvalues are equal. Only one component of $\bar{A}_{a b}$ is independent and an experiment with one (arbitrary) direction of excitation $\boldsymbol{E}$ yields all available information. One eigendirection of the tensor of emission lies parallel to $\boldsymbol{E}$. The corresponding eigenvalue is usually called $I_{\|}$. From the assumption of isotropy follows that the intensity of emission is axially symmetric with $\vartheta=0$ as axis of symmetry, i.e. the two eigenvalues of $\bar{I}_{a b}$, belonging to the eigendirections perpendicular to $\boldsymbol{E}$, are equal. Usually they are called $I_{\perp}$. Hence in two independent components $I_{\|}, I_{\perp}$ of $\bar{I}_{a b}$ and therefore of $F_{a b c d}$ all available information is contained.

With $I_{\|}, I$ the degree of polarization $P$

$$
P=\left(I_{\|}-I_{\perp}\right) /\left(I_{\|}+I_{\perp}\right)
$$

may be defined, which is independent of the absolute intensities.

\section{Discussion}

As we have seen, by the static polarized fluorescence experiment the multipole moments of the 
orientation distribution of absorption moments can be determined up to the fourth (hexadecapole) moments if the transition moments of absorption and emission are parallel. In the general case of arbitrary rearrangement at least the information up to the second (quadrupole) moments is contained in the fluorescence tensor $F_{a b c d}$, because according to (17 a) the tensor of absorption $\bar{A}_{a b}$ is uniquely given by $F_{a b c d}$. Therefore $\overline{A_{a b}}$ may be determined by the polarized absorption experiment as well as by fluorescence polarization measurements. Generally fluorescence polarization yields additional information about rearrangement and orientation. But a general statement that from $F_{a b c d}$ the orientation distribution may be determined up to the hexadecapole moments, is not possible. There are simple theoretical examples, where all information about higher multipoles than the quadrupole is lost during the rearrangement: When the transition moments are equipartitioned over the unit sphere during the excited state, the emission is isotropic. Obviously this means that $F_{a b c d}=0$ for $a \neq b$ and

$$
F_{x x c d}=F_{y y c d}=F_{z z c d}=\frac{1}{3} \overline{A_{c d}} \text {. }
$$

Therefore in $F_{a b c d}$ is contained not more information about $F_{\mathrm{A}}$ than in $\bar{A}_{a b}$. In addition, of course, important information about the process of rearrangement is obtained.

1 F. Perrin, J. Phys. Rad. 7, 930 [1926].

2 F. Perrin, Ann. Phys., Paris 10, 169 [1929].

3 F. Perrin, J. Phys. Radium 7, 1 [1936].

${ }^{4}$ R. Memming, Z. Phys. Chem. 28, 168 [1961].

5 J. R. Lombardi and G. A. Dafforn, J. Chem. Phys. 44, 3882 [1966].

6 T. Tao, Biopolymers 8, 609 [1969].

7 R. D. Spencer and G. Weber, J. Chem. Phys. 52, 1654 [1970].

8 G. Weber, J. Chem. Phys. 55, 2399 [1971].

9 Ph. Wahl, C. R. Acad. Sci. Paris 263, 1525 [1966].

10 T. J. Chuang and K. B. Eisenthal, J. Chem. Phys. 57, 5094 [1972].

11 Y. Nishijima, Y. Onogi, and T. Asai, J. Polymer Sci. Part C 15, 237 [1965].
The whole formalism used in this paper may be applied also for the time-dependent fluorescence polarization experiment. Instead of $F\left(\vartheta, \varphi, \vartheta^{\prime}, \varphi^{\prime}\right)$ one has to introduce a time-dependent function $\stackrel{t}{F}\left(\vartheta, \varphi, \vartheta^{\prime}, \varphi^{\prime}, t\right)$, which is defined as follows: if transition moments of absorption in orientation between $(\vartheta, \varphi)$ and $(\vartheta+\mathrm{d} \vartheta, \varphi+\mathrm{d} \varphi)$ are excited at time $t=0$ then with a probability proportional to $\stackrel{t}{F}\left(\vartheta, \varphi, \vartheta^{\prime}, \varphi^{\prime}, t\right) \mathrm{d} \Omega \mathrm{d} \Omega^{\prime} \mathrm{d} t$ this excitation induces an emission in the time interval $[t, t+\mathrm{d} t]$ by transition moments in orientation between $\left(\vartheta^{\prime}, \varphi^{\prime}\right)$ and $\left(\vartheta^{\prime}+\mathrm{d} \vartheta^{\prime}, \varphi^{\prime}+\mathrm{d} \varphi^{\prime}\right)$. The resulting fluorescence tensor $\stackrel{t}{F}_{a b c d}$ is now time dependent and determines the polarized fluorescence intensities measured at time $t$, when the probe is excited by a short light pulse at time $t=0$. Measurements of the time dependence of $\stackrel{t}{F}_{a b c d}$ yield important information on the processes determining the rearrangement of transition moments (e.g. coefficients of rotational diffusion), if their typical relaxation times are comparable with the fluorescence life-time.

\section{Acknowledgement}

I thank Prof. P. Läuger for discussions and critically reading the manuscript.

12 Y. Nishijima, J. Polymer Sci. Part C 31, 353 [1970].

13 C. R. Desper and J. Kimura, J. Appl. Phys. 38, 4225 [1967].

14 R. A. Badley, H. Schneider, and W. G. Martin, Biochem. Biophys. Res. Commun. 45, 174 [1971].

15 R. A. Badley, W. G. Martin, and H. Schneider, Biochemistry 12, 268 [1973].

16 J. Yguerabide and L. Stryer, Proc. Nat. Acad. Sci. 68, 1217 [1971].

17 E. Frehland and H.-W. Trissl, J. Membrane Biol. 21, 147 [1975].

18 E. Klingbeil, "Tensorrechnung für Ingenieure", Bibliographisches Institut Mannheim 1966. 